\title{
Chirped Optical Solitons in Birefringent Fibers with Parabolic Law Nonlinearity and Four-Wave Mixing
}

\author{
H. TRIKI ${ }^{a}$, A. Biswas ${ }^{b}$, D. Milović ${ }^{c}$ AND M. BeliĆ ${ }^{d}$ \\ ${ }^{a}$ Radiation Physics Laboratory, Department of Physics, Faculty of Sciences, Badji Mokhtar University, \\ P.O. Box 12, 23000 Annaba, Algeria \\ ${ }^{b}$ Department of Mathematical Science, Delaware State University, Dover, DE 19901-2277, USA \\ and Faculty of Science, Department of Mathematics, King Abdulaziz University, Jeddah-21589, Saudi Arabia \\ ${ }^{c}$ Faculty of Electronic Engineering, Department of Telecommunications, University of Nis, \\ Aleksandra Medvedeva 14, 18000 Nis, Serbia \\ ${ }^{d}$ Science Program, Texas A\&M University at Qatar, P.O. Box 23874, Doha, Qatar
}

(Received March 23, 2016)

\begin{abstract}
We investigate exact soliton solutions with nonlinear chirp for the coupled nonlinear Schrödinger equations with cubic-quintic nonlinearity, self-steepening, self-frequency shift and four-wave mixing. The model governs the femtosecond pulse propagation in birefringent fibers. We introduce a new ansatz to obtain the nonlinear chirp associated with the propagating soliton pulses. New chirped soliton pair solutions with non-trivial chirping are found for the coupled nonlinear equations, illustrating the potentially rich set of solitonic pulse solutions of the model with higher-order effects. The solutions comprise two types of bright-W-shaped and bright-bright soliton pairs as well as kink and anti-kink pulses. Interestingly, the bright wave in the bright-W shaped soliton pairs possesses a platform underneath it, originating from the self-steepening and self-frequency shift effects. The corresponding chirp associated with each of these optical soliton pairs is also determined. It is shown that the nonlinear chirp is related to the pair intensity and determined by self-frequency shift and pause self-steepening. Parametric conditions for the existence and uniqueness of chirped solutions are given.
\end{abstract}

DOI: 10.12693/APhysPolA.130.718

PACS/topics: 42.81.Dp, 42.65.Tg

\section{Introduction}

Soliton propagation in nonlinear media is an important subject that has attracted much interest in many fields of physics such as nonlinear optics [1,2], plasma physics [3], and nonlinear quantum field theory [4]. In a singlemode optical fiber, the soliton dynamics is described by the nonlinear Schrödinger (NLS) equation for a scalar field. It is commonly known that the NLS equation admits bright and dark soliton-type solutions in anomalous and normal dispersion regimes, respectively. Soliton can propagate over a long distance without the amplitude attenuation and shape change in the uniform nonlinear fiber under the condition that the group velocity dispersion (GVD) balances the self-phase modulation [5]. However, modeling the propagation of ultrashort (femtosecond) optical pulses cannot be described by using of the NLS model. It has been demonstrated that when the pulses are shorter than $100 \mathrm{fs}$, the higher order effects in nonlinear media become important, and therefore the governing equation should still include third-order dispersion (TOD), the self-steepening (SS), and the selffrequency shift [6]. The effect of TOD is significant for fs pulses when the GVD is close to zero [7]. It is negligible for optical pulses whose width is of the order of $100 \mathrm{fs}$ or more, having power of the order of $1 \mathrm{~W}$ and GVD far away from zero [7]. The effect of SS is due to intensity dependent group velocity of the optical pulse, which gives the pulse a very narrow width in the course of propagation [8]. The stimulated Raman scattering is due to the delayed response of the medium, which forces the pulse to undergo a frequency shift, known as self-frequency shift [8]. Notice that various types of exact solitons or solitary wave solutions of higher-order NLS-type equations have been studied extensively, both theoretically and numerically [9-17]. In particular, a new form of solitary wave solution that takes the shape of $\mathrm{W}$ was found for the first time for a single higher-order NLS equation with third-order dispersions, self-steepening, and selffrequency shift effects by Li et al. [18]. Another novel localized solution named dipole soliton, dubbed "darkin-the-bright" solitary wave solution has been recently found for a higher-order NLS equation with non-Kerr nonlinearity in Ref. [19]. Such solution is obtained using a soliton ansatz solution composed of the product of bright and dark solitary waves.

For applications for which polarization effects are important, one must consider a system of coupled NLS equations that is generally not integrable [20]. Such nonlinear models possess applications in the study of soliton wavelength division multiplexing, soliton switching in the birefringent optical fibers, multichannel bit parallelwavelength optical-fiber networks, propagation and collision of the temporal vector solitons in birefringent fibers, etc. [21]. In realistic optical fibers, no mode is single due to the presence of birefringence. Indeed, single mode fibers are bimodal $[8,22]$.

In recent years, considerable attention has been paid to the investigation of chirped femtosecond solitons 
propagating in nonlinear optical fibers [7, 23, 24]. Applications of such objects include pulse compression or amplification, optical pulse compressors, and solitary-wavebased communications links $[25,26]$. However, much of it is confined to propagating scalar fields that are described by a single higher-order NLS equation. To the best of our knowledge, vector soliton solutions with nonlinear chirping for coupled higher-order NLS models have not been uncovered as yet. Compared with that for the scalar NLS equation, the case for the coupled models will be more complicated due to the presence of nonlinear coupling terms.

In this paper we investigate for the first time to our knowledge soliton pairs with nonlinear chirp for a system of two coupled NLS equations describing the propagation of femtosecond optical pulses in a birefringent fiber lightguide. We have shown that the coupled NLS equations in the presence of the quintic nonlinearity, self-steepening, self-frequency shift and four-wave mixing term possesses rich solitonic solutions with non-trivial chirping. The soliton pairs comprise bright-W shaped, kink and anti-kink, and bright-bright solutions with a nonlinear chirp which is related to the pair intensity. The parameter domains in which these chirped solutions exist are given. The corresponding chirp associated with each of these localized solutions is also found.

This article is organized as follows. In Sect. 2, we present the coupled NLS equations considered and the nonlinear chirp ansatz that is used to determine the chirping associated with propagating envelope solutions. Then, we find exact families of soliton pair solutions for the basic model under consideration in Sect. 3. The corresponding chirp associated with each of these families is also determined. Parametric conditions for the existence of chirped solutions are given. Finally, we summarize our findings and give future directions of work in Sect. 4 .

\section{Model and equations}

Consider the following system governing the dynamics of the coherently coupled orthogonally polarized waveguide modes in the Kerr medium:

$$
\begin{aligned}
& \mathrm{i} q_{1 z}+\alpha q_{1 \tau \tau}+\left(\left|q_{1}\right|^{2}+\sigma\left|q_{2}\right|^{2}\right) q_{1}+\lambda q_{2}^{2} q_{1}^{*}=0, \\
& \mathrm{i} q_{2 z}+\alpha q_{2 \tau \tau}+\left(\sigma\left|q_{1}\right|^{2}+\left|q_{2}\right|^{2}\right) q_{2}+\lambda q_{1}^{2} q_{2}^{*}=0,
\end{aligned}
$$

which covers systems (1), (3), and (4) given in Ref. [21]. Here $q_{1}$ and $q_{2}$ are the complex amplitudes in both polarization modes, $\sigma$ is the incoherent coupling parameter (the cross-phase modulation as well), $\alpha$ is the parameter of GVD, $\lambda$ is the coherent coupling parameter (the four-wave mixing as well), and * denotes the complex conjugate. Note that the coherent coupling governs the energy exchange between two axes of the fiber [21].

As a natural generalization of the above model with the cubic nonlinearity, we consider the following model of coupled NLS-type equations with quintic nonlinearity, self-steepening and self-frequency shift terms:

$$
\begin{aligned}
& \mathrm{i} q_{1 z}+\alpha q_{1 \tau \tau}+s\left(\left|q_{1}\right|^{2}+\sigma\left|q_{2}\right|^{2}\right) q_{1}+\lambda q_{2}^{2} q_{1}^{*} \\
& \quad+\mathrm{i} \gamma\left[\left(\left|q_{1}\right|^{2}+\eta\left|q_{2}\right|^{2}\right) q_{1}\right]_{\tau}+\mathrm{i} \delta q_{1}\left(\left|q_{1}\right|^{2}+r\left|q_{2}\right|^{2}\right)_{\tau} \\
& \quad+\theta\left(\left|q_{1}\right|^{2}+\Gamma\left|q_{2}\right|^{2}\right)^{2} q_{1}=0, \\
& \mathrm{i} q_{2 z}+\alpha q_{2 \tau \tau}+s\left(\sigma\left|q_{1}\right|^{2}+\left|q_{2}\right|^{2}\right) q_{2}+\lambda q_{1}^{2} q_{2}^{*} \\
& \quad+\mathrm{i} \gamma\left[\left(\eta\left|q_{1}\right|^{2}+\left|q_{2}\right|^{2}\right) q_{2}\right]_{\tau}+\mathrm{i} \delta q_{2}\left(r\left|q_{1}\right|^{2}+\left|q_{2}\right|^{2}\right)_{\tau} \\
& \quad+\theta\left(\Gamma\left|q_{1}\right|^{2}+\left|q_{2}\right|^{2}\right)^{2} q_{2}=0 .
\end{aligned}
$$

where $\gamma, \delta$ and $\theta$ are the coefficients of the selfsteepening, self-frequency shift, and quintic nonlinearity, respectively.

In the limiting case $\gamma=\delta=\theta=0$ and $s=1$, the system of Eqs. (3) and (4) transforms into the Kerr model of Eqs. (1) and (2) describing the orthogonally polarized optical waves propagation in a cubic anisotropic nonlinear medium. Notice that a more general form of coupled nonlinear Schrödinger equation with cubic-quintic nonlinearity (3) and (4) involving the linear coupling terms, thirdorder dispersion and other additional terms was proposed by Radhakrishnan et al. [27]. In Ref. [28], the one-, two- and three-soliton solutions have been derived for the generalized coupled nonlinear Schrödinger equations with cubic-quintic nonlinearity based on the bilinear form obtained via the dependent variable transformation and the Hirota method. Furthermore, the Darboux transformation and soliton solutions for the coupled nonlinear Schrödinger-typed equations with cubic-quintic nonlinear terms have been recently given [29]. In absence of quintic nonlinearity and when the effect of third order dispersion is included and the four-wave mixing term is replaced by another kind of the Raman contribution, the unchirped cnoidal and solitary wave solutions of the coupled higher order NLS equations have been investigated by using coupled amplitude-phase formulation [8].

The most challenging problem is to find various types of exact soliton solutions with nonlinear chirp to Eqs. (3) and (4). Such a problem is very important in understanding widely different nonlinear phenomena arising in birefringent optical fibers. In the present work, we have been able to find five families of chirped soliton solutions for the model under consideration, together with the conditions that specify the parameter domains in which they exist. Moreover, the nonlinear chirp associated with each of these solitonic pairs is also determined.

To find chirped soliton solutions of the above model, we firstly generalize the complex envelope traveling-wave solutions used in Ref. [23] to the case of two soliton modes as

$$
\begin{aligned}
& q_{1}(z, \tau)=A(\xi) \mathrm{e}^{\mathrm{i}(\chi(\xi)-k z)}, \\
& q_{2}(z, \tau)=B(\xi) \mathrm{e}^{\mathrm{i}(\chi(\xi)-k z)},
\end{aligned}
$$

where $A, B$ and $\chi$ are real functions of the traveling coordinate $\xi=\tau-u z$. Here $u$ is given in term of the group velocity of the wave packet as $u=1 / v$. The corresponding chirp is given by $\delta \omega(\tau, z)=-\frac{\partial}{\partial \tau}(\chi(\xi)-k z)=-\chi^{\prime}(\xi)$. 
Substituting the solutions (5) and (6) into Eq. (3) and separating the real and imaginary parts leads to

$$
\begin{aligned}
& k A+u \chi^{\prime} A+\alpha A^{\prime \prime}-\alpha \chi^{\prime 2} A+s A^{3}+(s \sigma+\lambda) A B^{2}-\gamma \chi^{\prime} A^{3} \\
& \quad-\gamma \eta \chi^{\prime} A B^{2}+\theta\left(A^{6}+\Gamma^{2} A B^{4}+2 \Gamma A^{3} B^{2}\right)=0 \\
& -u A^{\prime}+2 \alpha A^{\prime} \chi^{\prime}+\alpha A \chi^{\prime \prime}+(3 \gamma+2 \delta) A^{2} A^{\prime} \\
& \quad+2(\gamma \eta+\delta r) A B B^{\prime}+\gamma \eta B^{2} A^{\prime}=0 .
\end{aligned}
$$
and

Now inserting the solutions (5) and (6) into Eq. (4) and separating the real and imaginary parts leads to

$$
\begin{gathered}
k B+u \chi^{\prime} B+\alpha B^{\prime \prime}-\alpha \chi^{\prime 2} B+s B^{3}+(s \sigma+\lambda) A^{2} B-\gamma \chi^{\prime} B^{3} \\
-\gamma \eta \chi^{\prime} A^{2} B+\theta\left(B^{6}+\Gamma^{2} A^{4} B+2 \Gamma A^{2} B^{3}\right)=0,
\end{gathered}
$$

and

$$
\begin{gathered}
-u B^{\prime}+2 \alpha B^{\prime} \chi^{\prime}+\alpha B \chi^{\prime \prime}+(3 \gamma+2 \delta) B^{2} B^{\prime} \\
+2(\gamma \eta+\delta r) A A^{\prime} B+\gamma \eta A^{2} B^{\prime}=0 .
\end{gathered}
$$

To solve the set of Eqs. (7) - (10), we introduce the ansatz

$$
\chi^{\prime}=p\left(A^{2}+B^{2}\right)+q,
$$

where $p$ is the nonlinear chirp parameter and $q$ is the constant chirp parameter. Accordingly, the resultant chirp consisting of linear and nonlinear contributions can be obtained as

$$
\delta \omega(\tau, z)=-p\left(A^{2}+B^{2}\right)-q .
$$

The substitution of ansatz (11) into Eq. (8) and (10) yields four algebraic equations that define the chirp parameters:

$$
p=-\frac{3 \gamma+2 \delta}{4 \alpha}, \quad q=\frac{u}{2 \alpha}
$$

and give important constraint equations between the model coefficients as $\gamma \eta+2 \delta r=0$ and $3 \gamma+2 \delta=2 \gamma \eta$. This implies that the parameters $\delta, \eta, r$ and $\gamma$ are not independent and the propagating chirped soliton pairs are obtained in the framework of these conditions.

It is very interesting to see that the phase in (11) has a nontrivial form and has an intensity dependent chirping term related to the pair intensity $I=\left|q_{1}\right|^{2}+\left|q_{2}\right|^{2}=$ $A^{2}+B^{2}$, in addition to the linear contribution described by the last term. Note that one must require $p \neq 0$ in (12) in order to obtain nonlinearly chirped soliton pair solutions for Eqs. (3) and (4). In order to ensure this we select appropriate self-steepening and self-frequency shift coefficients in (13) so that $3 \gamma+2 \delta \neq 0$. In the trivial limit, when $p=0$, the expression (12) becomes independent of the amplitudes $A$ and $B$, and the resulting soliton solutions will present a linear chirp.

Now using Eqs. (11) and (13) in Eqs. (7) and (9), we respectively obtain

$$
\begin{aligned}
& A^{\prime \prime}-a_{1} A^{6}+a_{2} A^{3}+a_{3} A B^{2}-a_{4} A^{3} B^{2} \\
& -a_{6} A B^{4}-a_{6} A=0,
\end{aligned}
$$

and

$$
\begin{aligned}
& B^{\prime \prime}-a_{1} B^{6}+a_{2} B^{3}+a_{3} B A^{2}-a_{4} B^{3} A^{2} \\
& \quad-a_{6} B A^{4}-a_{6} B=0,
\end{aligned}
$$

where

$$
\begin{aligned}
a_{1}= & \frac{(2 \delta+3 \gamma)(2 \delta-\gamma)-16 \alpha \theta}{16 \alpha^{2}}, \quad a_{2}=\frac{2 \alpha s-u \gamma}{2 \alpha^{2}}, \\
a_{3} & =\frac{2 \alpha(\sigma s+\lambda)-u \gamma \eta}{2 \alpha^{2}}, \\
a_{4} & =\frac{(2 \delta+3 \gamma)(2 \delta+\gamma-2 \gamma \eta)-16 \alpha \theta \Gamma}{8 \alpha^{2}}, \\
a_{6} & =\frac{(2 \delta+3 \gamma)(2 \delta+3 \gamma-4 \gamma \eta)-16 \alpha \theta \Gamma^{2}}{16 \alpha^{2}}, \\
a_{6} & =-\frac{4 k \alpha+u^{2}}{4 \alpha^{2}} .
\end{aligned}
$$

Equations (14) and (15) are elliptic-type differential equations with a fifth-degree nonlinear term. In what follows, we present, for the first time to our knowledge, new types of soliton pair solutions with nonlinear chirp for these equations. We also report parametric conditions for which these optical solitons exist.

\section{Chirped soliton solutions pairs}

We note that the finding of exact soliton solutions to Eqs. (14) and (15) is a nontrivial issue. Below we propose efficient ansätze which are able to determine various chirped solitonic solutions for the above equations. By considering such ansätze solution, we can offer an accurate estimate of the dynamics of chirped solitons in birefringent fibers such as their rules of evolution and conditions of existence. Here we have found five types of soliton pair solutions for these coupled equations. Interestingly, such solutions are derived in the most general case, when all the coefficients $a_{i}$ (with $i=1, \ldots, 6$ ) have nonzero values.

\subsection{Case $I$}

To start with, we propose localized soliton ansätze of the type

$$
\begin{aligned}
& A(\xi)=\rho+w \sqrt{\operatorname{sech}(\mu \xi)}, \\
& B(\xi)=\rho-w \sqrt{\operatorname{sech}(\mu \xi)},
\end{aligned}
$$

where $\rho, w$ and $\mu$ are unknown parameters to be determined.

Substituting expressions (17) and (18) into Eqs. (14) and (15) and equating the equal powers of $\operatorname{sech}(\mu \xi)$ to zero yields the following consistency conditions:

$$
\begin{aligned}
& -\left(a_{1}+a_{4}+a_{6}\right) \rho^{6}+\left(a_{2}+a_{3}\right) \rho^{3}-a_{6} \rho=0, \\
& \frac{1}{4} w \mu^{2}-\left(5 a_{1}+a_{4}-3 a_{6}\right) \rho^{4} w \\
& \quad+\left(3 a_{2}-a_{3}\right) \rho^{2} w-a_{6} w=0 \\
& w^{3}\left(-10 a_{1} \rho^{2}+a_{2}+a_{3}+2 a_{4} \rho^{2}-2 a_{6} \rho^{2}\right)=0, \\
& \frac{3}{4} w \mu^{2}+\left(a_{1}+a_{4}+a_{6}\right) w^{6}=0 \\
& w^{2} \rho\left(-10 a_{1} \rho^{2}+3 a_{2}-a_{3}+2 a_{4} \rho^{2}-2 a_{6} \rho^{2}\right)=0 \\
& \rho w^{4}\left(5 a_{1}+a_{4}-3 a_{6}\right)=0 .
\end{aligned}
$$


First, one solves Eq. (24) to obtain a necessary condition for the existence of the above soliton pair solutions as: $5 a_{1}+a_{4}-3 a_{6}=0$. Second, using Eqs. (21) and (23), we find another important constraint equation which must be satisfied: $a_{2}=a_{3}$. Introducing these conditions into Eqs. (20) - (23), one obtains the soliton parameters $\rho, \mu$ and $w$ as

$$
\begin{aligned}
\rho & =\sqrt{\frac{a_{2}}{4 a_{6}-2 a_{4}}}, \\
\mu & =2 \sqrt{\frac{a_{6}\left(2 a_{6}-a_{4}\right)-a_{2}^{2}}{2 a_{6}-a_{4}}}, \\
w & =\sqrt[4]{\frac{3 a_{2}^{2}-3 a_{6}\left(2 a_{6}-a_{4}\right)}{\left(2 a_{6}-a_{4}\right)\left(a_{1}+a_{4}+a_{6}\right)}} .
\end{aligned}
$$

Also from relations (25) and (19) the following restriction is obtained: $a_{2}^{2}\left(7 a_{6}-5 a_{4}-a_{1}\right)-4 a_{6}\left(2 a_{6}-a_{4}\right)^{2}=0$. Now because parameter $\mu$ needs to be positive for the existence of the solutions (17) and (18), one must choose the parameters $a_{i}$ to satisfy $\left(2 a_{6}-a_{4}\right)\left[a_{6}\left(2 a_{6}-a_{4}\right)-a_{2}^{2}\right]>$ 0 , as it follows from (26). We also find from (25) that one must require $a_{2}\left(2 a_{6}-a_{4}\right)>0$ for the parameter $\rho$ to exist.

If we insert the solutions (17) and (18) into (5) and (6), we obtain new chirped soliton solutions of Eqs. (4) and (5) of the form

$$
\begin{aligned}
& q_{1}(z, \tau)=[\rho+w \sqrt{\operatorname{sech}(\mu(\tau-u z))}] \mathrm{e}^{\mathrm{i}(\chi(\xi)-k z),} \\
& q_{2}(z, \tau)=[\rho-w \sqrt{\operatorname{sech}(\mu(\tau-u z))}] \mathrm{e}^{\mathrm{i}(\chi(\xi)-k z,}
\end{aligned}
$$

which exist provided that the conditions: $\gamma \eta+2 \delta r=0$, $3 \gamma+2 \delta=2 \gamma \eta, 5 a_{1}+a_{4}-3 a_{6}=0, a_{2}=a_{3}$, and $a_{2}^{2}\left(7 a_{6}-5 a_{4}-a_{1}\right)-4 a_{6}\left(2 a_{6}-a_{4}\right)^{2}=0$ are satisfied.

Figure 1a and b depicts intensity profiles of the above soliton pair for the following values of the model parameters: $\alpha=1.6001, s=-2.6885, \sigma=1, \gamma=0.30814$, $\eta=3.98601, \delta=0.76604, r=-0.801687, \theta=0.235207$, $u=4.1184, k=-39.431$ and $\Gamma=\frac{2}{3}$. To make $a_{2}=a_{3}$, we set $\lambda=1.1841064$. This leads to a bright-type soliton solution $\left|q_{1}(z, \tau)\right|^{2}$ that has a pronounced platform underneath it, and an interesting envelope solution $\left|q_{2}(z, \tau)\right|^{2}$ that takes the shape of W. Clearly, the intensity profile of the $q_{2}$-soliton is similar with the $\mathrm{W}$-shaped soliton reported in [18]. However, the solution form is distinctive from the ones in [18] in having the $\sqrt{\text { sech- }}$ shaped envelope.

It is also very interesting to see that Eqs. (14) and (15) possess another type of bright-W shaped soliton pair of the form

$$
\begin{aligned}
& A(\xi)=\rho+w \sqrt{\operatorname{sech}(\mu \xi)}, \\
& B(\xi)=-\rho+w \sqrt{\operatorname{sech}(\mu \xi)},
\end{aligned}
$$

which has the same parameters as those given in Eqs. (25) $-(27)$.

The corresponding associated chirping is obtained by substituting the solutions (17) and (18) into Eq. (12) as
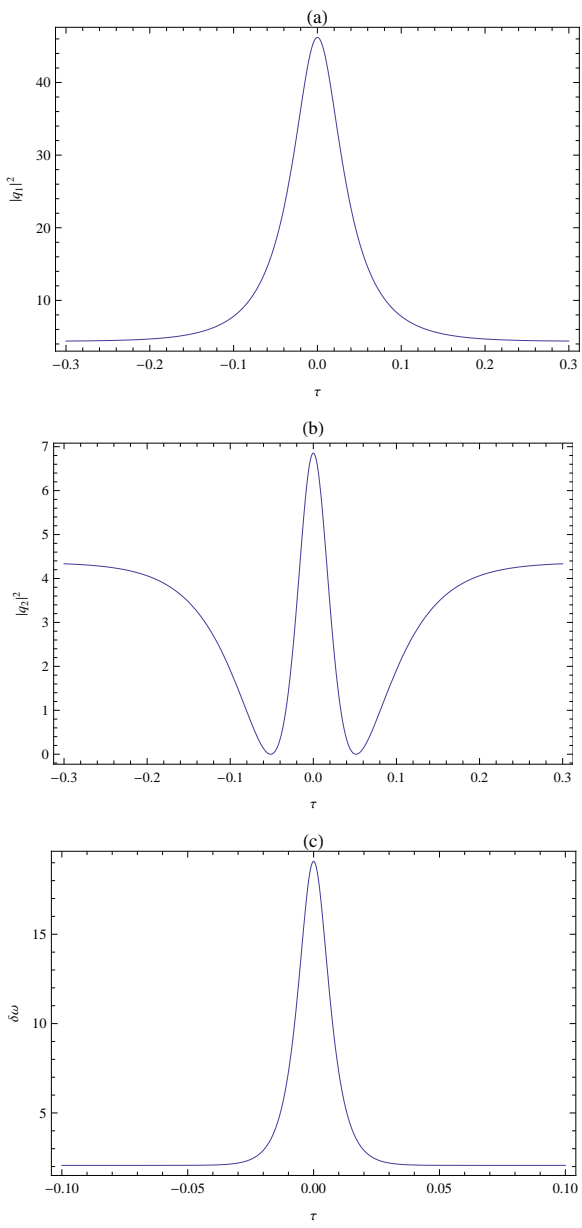

Fig. 1. Intensity profile for the (a) bright soliton $\left|q_{1}(0, \tau)\right|^{2}$ as a function of $\tau$ as computed from Eq. (28) and (b) W-shaped soliton $\left|q_{2}(0, \tau)\right|^{2}$ as computed from Eq. (29). (c) Chirping profile of soliton pair as a function of $\tau$ as computed from Eq. (32). The parameters are given in the text.

$$
\delta \omega(\tau, z)=-2 p\left(\rho^{2}+w^{2} \operatorname{sech}(\mu \xi)\right)-q,
$$

where the first two terms denote the nonlinear chirp that results from the self-steepening and self-frequency shift, while the last term accounts for the linear chirp. Figure $1 \mathrm{c}$ shows the resultant chirping (32) at $z=0$.

\subsection{Case II}

Now we propose the following soliton ansätze for solving Eqs. (14) and (15) :

$$
\begin{aligned}
& A(\xi)=f+g \operatorname{sech}(\mu \xi), \\
& B(\xi)=f-g \operatorname{sech}(\mu \xi),
\end{aligned}
$$

where $f, g$ and $\mu$ are real constants to be determined by the physical parameters of the model.

Substituting expressions (33) and (34) into Eqs. (14) and (15) and equating coefficients of the same powers of $\operatorname{sech}^{j}(\mu \xi)$ for $j=0, . ., 5$ to zero, we obtain

$$
\begin{aligned}
& -\left(a_{1}+a_{4}+a_{6}\right) f^{6}+\left(a_{2}+a_{3}\right) f^{3}-a_{6} f=0, \\
& -g \mu^{2}+\left(5 a_{1}+a_{4}-3 a_{6}\right) f^{4} g
\end{aligned}
$$




$$
\begin{aligned}
& \quad+\left(a_{3}-3 a_{2}\right) f^{2} g-a_{6} g=0, \\
& f g^{2}\left(-10 a_{1} f^{2}+3 a_{2}-a_{3}+2 a_{4} f^{2}-2 a_{6} f^{2}\right)=0, \\
& 2 g \mu^{2}+10 a_{1} f^{2} g^{3}-a_{2} g^{3}-a_{3} g^{3}-2 a_{4} f^{2} g^{3} \\
& \quad+2 a_{6} f^{2} g^{3}=0, \\
& f g^{4}\left(5 a_{1}+a_{4}-3 a_{6}\right)=0, \\
& g^{6}\left(a_{1}+a_{4}+a_{6}\right)=0,
\end{aligned}
$$

Equations (39) and (40) can be solved to yield the necessary conditions for the existence of soliton solutions (33) and (34) as: $a_{4}+2 a_{6}=0$, and $a_{1}=a_{6}$. Inserting the condition (40) into Eq. (35), we obtain

$$
f=\sqrt{\frac{a_{6}}{a_{2}+a_{3}}} .
$$

Now substituting the condition (39) and Eq. (41) into Eq. (36), one can obtain the pulse width $\mu$ as

$$
\mu=\sqrt{\frac{2 a_{6}\left(a_{3}-a_{2}\right)}{a_{2}+a_{3}} .}
$$

By using (39) and (42), Eq. (38) gives

$$
g=\sqrt{\frac{2 a_{6}}{a_{2}+a_{3}}} .
$$

From these expressions, we can see that one must choose the parameters $a_{2}, a_{3}$ and $a_{6}$ to satisfy $a_{6}\left(a_{2}+a_{3}\right)>0$ and $a_{3}>a_{2}$. Having obtained the expressions for the pulse parameters $f, g$ and $\mu$, we construct a new family of chirped soliton pair solutions for Eqs. (14) and (15) as follows:

$$
\begin{aligned}
& q_{1}(z, \tau)=[f+g \operatorname{sech}(\mu(\tau-u z))] \mathrm{e}^{\mathrm{i}(\chi(\xi)-k z)}, \\
& q_{2}(z, \tau)=[f-g \operatorname{sech}(\mu(\tau-u z))] \mathrm{e}^{\mathrm{i}(\chi(\xi)-k z)} .
\end{aligned}
$$

which exist provided all the previous conditions are satisfied [namely, $\gamma \eta+2 \delta r=0,3 \gamma+2 \delta=2 \gamma \eta, a_{4}+2 a_{6}=0$, $a_{1}=a_{6}, a_{6}\left(a_{2}+a_{3}\right)>0$ and $\left.a_{3}>a_{2}\right]$.

The intensity profiles of this kind of soliton pair are shown in Fig. 2a and b. Here we have used the values: $\alpha=1.6001, \gamma=0.30814, \delta=0.15407, \eta=2, \Gamma=3$, $s=2.6885, \sigma=1, r=-2, \theta=-0.0074175, u=4.1184$, $\lambda=1.1841064$ and $k=-8.0214725$. As seen in these figures, the solutions (44) of $q_{1}$ describes the bright-type localized wave that has a pronounced platform underneath it, while the solution (45) accounts for the W-shaped profile of the wave $q_{2}$.

Here the wave for $q_{2}$ has the same sech solution form as for the $\mathrm{W}$-shaped soliton presented in [18]. But the intensity profile form is distinctive from the one in [18] which has an intensity value much higher in the middle than the one obtained in our case. It should be noticed that this intensity depends on the chosen parameter values which must satisfy the necessary conditions for the existence of the soliton solutions.

The chirp associated with these optical pulses is given by

$$
\delta \omega(\tau, z)=-2 p\left(f^{2}+g^{2} \operatorname{sech}^{2}(\mu \xi)\right)-q .
$$

Figure 2c presents the typical profile of chirping at $z=0$ for the same model parameters. As seen from the figure, the chirping for this soliton pair has a maximum at the center of the pulse and it saturates at a given finite value as $\tau \rightarrow \pm \infty$.
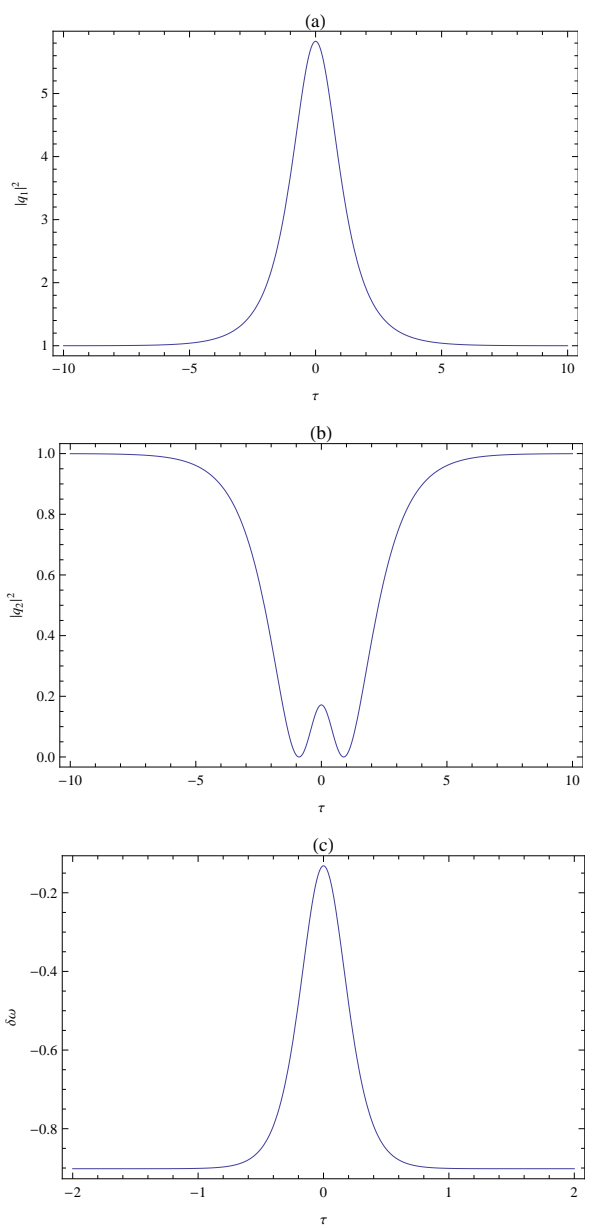

Fig. 2. Intensity profile for the (a) bright soliton $\left|q_{1}(0, \tau)\right|^{2}$ as a function of $\tau$ as computed from Eq. (44) and (b) W-shaped soliton $\left|q_{2}(0, \tau)\right|^{2}$ as computed from Eq. (45). (c) Chirping profile of soliton pair as a function of $\tau$ as computed from Eq. (46). The parameters are given in the text.

\subsection{Case III}

Next we investigate bright-type localized waves using the ansätze [30]:

$$
\begin{aligned}
& A(\xi)=b_{1} \operatorname{sech}(\mu \xi), \\
& B(\xi)=b_{2} \operatorname{sech}(\mu \xi),
\end{aligned}
$$

where $b_{1}$ and $b_{2}$ are real constants to be determined by the physical parameters of the model.

Substituting these ansätze into Eq. (14) and equating the coefficients of independent terms, one obtains

$$
\begin{aligned}
& b_{1}\left(\mu^{2}-a_{6}\right)=0, \\
& b_{1}\left(-2 \mu^{2}+a_{2} b_{1}^{2}+a_{3} b_{2}^{2}\right)=0, \\
& b_{1}\left(a_{1} b_{1}^{4}+a_{4} b_{1}^{2} b_{2}^{2}+a_{6} b_{2}^{4}\right)=0 .
\end{aligned}
$$


Now substituting these ansätze into Eq. (15) gives

$$
\begin{aligned}
& b_{2}\left(\mu^{2}-a_{6}\right)=0, \\
& b_{2}\left(-2 \mu^{2}+a_{2} b_{2}^{2}+a_{3} b_{1}^{2}\right)=0, \\
& b_{2}\left(a_{1} b_{2}^{4}+a_{4} b_{1}^{2} b_{2}^{2}+a_{6} b_{1}^{4}\right)=0 .
\end{aligned}
$$

The above set of equations can be readily solved to obtain the following expressions for the solitons parameters:

$$
\begin{aligned}
& \mu= \pm \sqrt{a_{6}}, \\
& b_{2}= \pm b_{1}= \pm \sqrt{\frac{2 a_{6}}{a_{2}+a_{3}}},
\end{aligned}
$$

and the condition: $a_{1}+a_{4}+a_{6}=0$. From these expressions it is obvious that $a_{6}>0$ and $a_{2}+a_{3}>0$ must be satisfied for the existence of the soliton pair solutions (47) and (48). By combining Eqs. (5), (6), (47) and (48), we obtain a chirped soliton pair solutions of the form

$$
\begin{aligned}
& q_{1}(z, \tau)=b_{1} \operatorname{sech}(\mu(\tau-u z)) \mathrm{e}^{\mathrm{i}(\chi(\xi)-k z)}, \\
& q_{2}(z, \tau)= \pm b_{1} \operatorname{sech}(\mu(\tau-u z)) \mathrm{e}^{\mathrm{i}(\chi(\xi)-k z)},
\end{aligned}
$$

which exist provided that $\gamma \eta+2 \delta r=0,3 \gamma+2 \delta=2 \gamma \eta$, $a_{1}+a_{4}+a_{6}=0, a_{6}>0$, and $a_{2}+a_{3}>0$.

Figure $3 \mathrm{a}$ and $\mathrm{b}$ display the intensity profiles of the soliton pair solutions $q_{1}$ and $q_{2}$ at $z=0$ for the following values of the model parameters: $\alpha=1.6001, s=-2.6885$, $\sigma=2, \gamma=0.30814, \eta=3.98601, \delta=0.76604, r=$ $-0.801687, \theta=-0.1174, u=-30.1280, k=-150.2856$, $\lambda=1$, and $\Gamma=0.4194$. These two solitons are brighttype pulses. We clearly see that the present solutions pair possess the same intensity profile in both components $q_{1}$ and $q_{2}$.

The substitution of the solutions (47) and (48) into Eq. (12) yields the chirp associated with the optical pulses

$$
\delta \omega(\tau, z)=-2 p b_{1}^{2} \operatorname{sech}^{2}(\mu \xi)-q .
$$

The corresponding pulse chirp obtained for the same values of parameters is shown in Fig. 3c.

\subsection{Case IV}

Let us now focus on kink type solitons using ansätze solution of the form [11]:

$$
\begin{aligned}
& A(\xi)=\varphi+\kappa \tanh (\mu \xi), \\
& B(\xi)=\varphi-\kappa \tanh (\mu \xi),
\end{aligned}
$$

where $\varphi$ and $\kappa$ are real constants to be determined by the physical parameters of the model.

Upon substituting these expressions into Eqs. (14) and (15) and collecting all the terms of the same power of $\operatorname{sech}^{i}(\mu \xi)$ and $\operatorname{sech}^{i}(\mu \xi) \tanh (\mu \xi)$ for $i=0,2$, 4, we can get the algebraic equations

$$
\begin{aligned}
& -a_{1} \varphi\left(\varphi^{4}+10 \varphi^{2} \kappa^{2}+5 \kappa^{4}\right)+a_{2} \varphi\left(\varphi^{2}+3 \kappa^{2}\right) \\
& \quad+a_{3} \varphi\left(\varphi^{2}-\kappa^{2}\right)-a_{4} \varphi\left(\varphi^{2}-\kappa^{2}\right)^{2} \\
& \quad-a_{6} \varphi\left(\varphi^{2}+3 \kappa^{2}\right)\left(\varphi^{2}-\kappa^{2}\right)-a_{6} \varphi=0 \\
& 10 a_{1} \varphi \kappa^{2}\left(\kappa^{2}+\varphi^{2}\right)+\left(a_{3}-3 a_{2}\right) \varphi \kappa^{2} \\
& \quad-2 a_{4} \varphi \kappa^{2}\left(\varphi^{2}-\kappa^{2}\right)-2 a_{6} \varphi \kappa^{2}\left(3 \kappa^{2}-\varphi^{2}\right)=0
\end{aligned}
$$
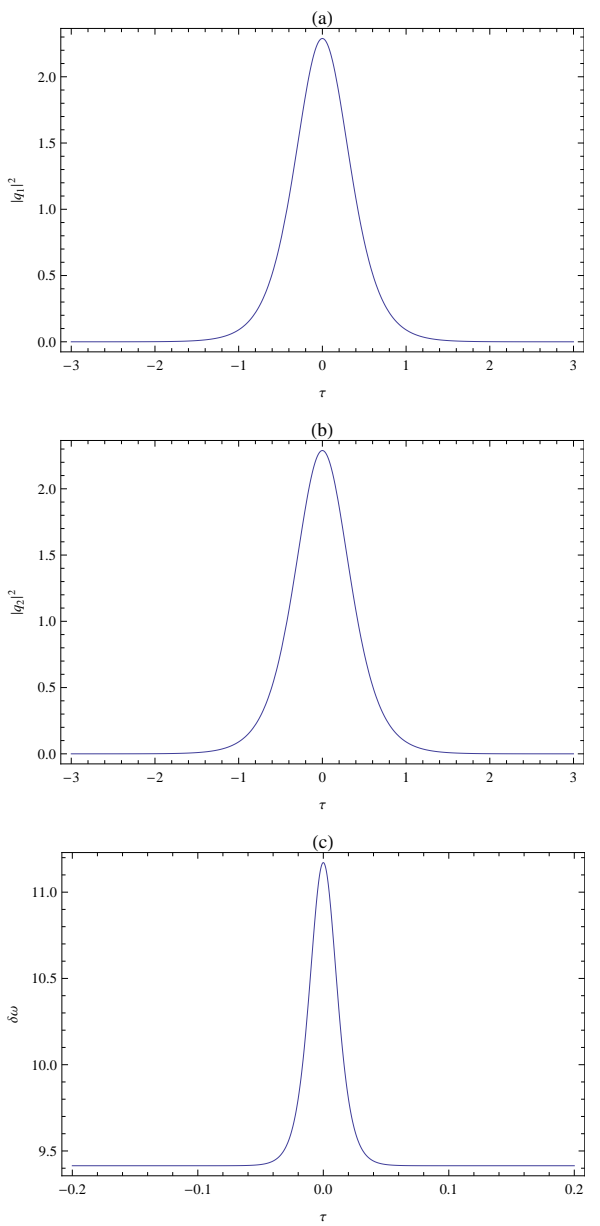

Fig. 3. Intensity profile for the (a) bright soliton $\left|q_{1}(0, \tau)\right|^{2}$ as a function of $\tau$ as computed from Eq. (57) and (b) bright soliton $\left|q_{2}(0, \tau)\right|^{2}$ as computed from Eq. (58). (c) Chirping profile of soliton pair as a function of $\tau$ as computed from Eq. (59). The parameters are given in the text.

$$
\begin{aligned}
& \varphi \kappa^{4}\left(5 a_{1}+a_{4}-3 a_{6}\right)=0, \\
& 2 \kappa \mu^{2}-2 a_{1} \kappa^{3}\left(\kappa^{2}+5 \varphi^{2}\right)+\left(a_{2}+a_{3}\right) \kappa^{3} \\
& \quad+2 a_{4} \kappa^{3}\left(\varphi^{2}-\kappa^{2}\right)-2 a_{6} \kappa^{3}\left(\kappa^{2}+\varphi^{2}\right)=0, \\
& \kappa^{6}\left(a_{1}+a_{4}+a_{6}\right)=0, \\
& a_{1} \kappa\left(\kappa^{4}+10 \varphi^{2} \kappa^{2}+5 \varphi^{4}\right)-a_{2} \kappa\left(\kappa^{2}+3 \varphi^{2}\right) \\
& \quad+a_{3} \kappa\left(\varphi^{2}-\kappa^{2}\right)+a_{4} \kappa\left(\varphi^{2}-\kappa^{2}\right)^{2} \\
& \quad+a_{6} \kappa\left(\kappa^{4}+2 \varphi^{2} \kappa^{2}-3 \varphi^{4}\right)+a_{6} \kappa=0 .
\end{aligned}
$$

Solving Eqs. (64) and (66) yield the necessary conditions for the existence of the present soliton pair solution: $a_{4}+2 a_{6}=0$ and $a_{1}=a_{6}$. If we insert these conditions into Eqs. (62) and (67), one gets $\varphi= \pm \kappa$. Substituting this latter relation into Eqs. (63), (65) and (67), we obtain

$$
\varphi= \pm \kappa=\frac{1}{4} \sqrt{\frac{3 a_{2}-a_{3}}{a_{1}}},
$$




$$
\mu=\frac{1}{4} \sqrt{\frac{\left(a_{2}-a_{3}\right)\left(3 a_{2}-a_{3}\right)}{a_{1}}},
$$

and the constraint of the system parameters: $\left(3 a_{2}-a_{3}\right)\left(a_{2}+a_{3}\right)=16 a_{1} a_{6}$. Notice that the pulse parameters $\varphi, \kappa$, and $\mu$ exist provided that $a_{1}\left(3 a_{2}-a_{3}\right)>0$ and $a_{2}>a_{3}$.

Substitution of the traveling wave solutions (60) and (61) in Eqs. (5) and (6) yields the following darkdark soliton pair solutions to Eqs. (3) and (4) :

$$
\begin{aligned}
& q_{1}(z, \tau)=[\varphi+\kappa \tanh (\mu(\tau-u z))] \mathrm{e}^{\mathrm{i}(\chi(\xi)-k z)}, \\
& q_{2}(z, \tau)=[\varphi-\kappa \tanh (\mu(\tau-u z))] \mathrm{e}^{\mathrm{i}(\chi(\xi)-k z)},
\end{aligned}
$$

which exist provided that the previous parametric conditions are satisfied [namely, $a_{1}=a_{6}, a_{4}+2 a_{6}=0$, $\left(3 a_{2}-a_{3}\right)\left(a_{2}+a_{3}\right)=16 a_{1} a_{6}, a_{1}\left(3 a_{2}-a_{3}\right)>0$ and $\left.a_{2}>a_{3}\right]$.
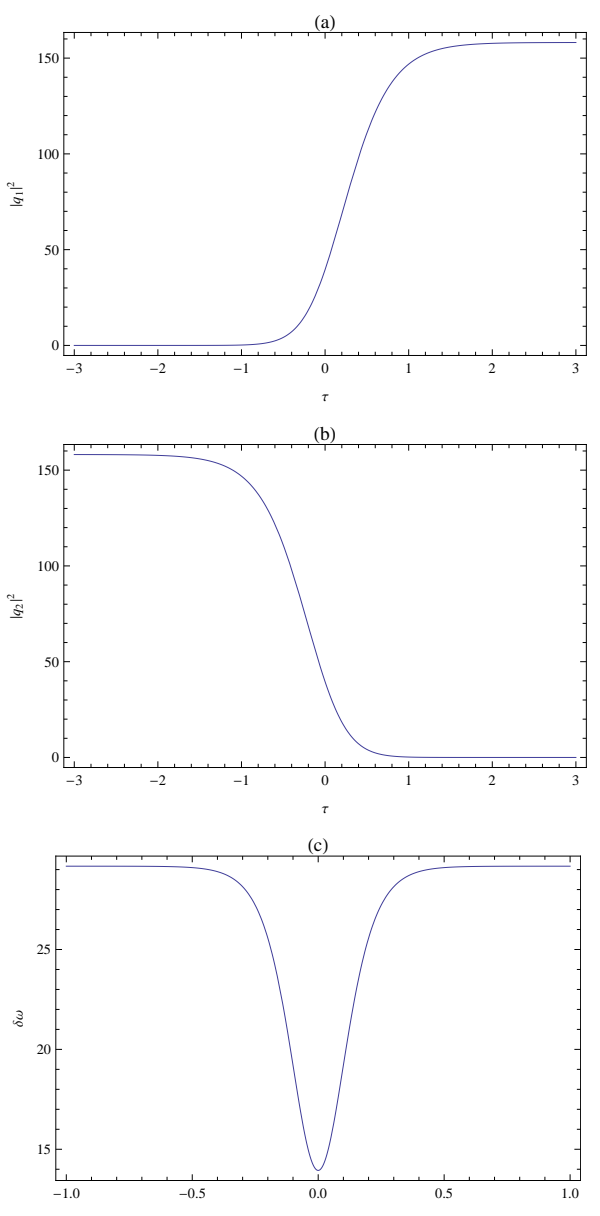

Fig. 4. Intensity profile for the (a) kink-shaped soliton $\left|q_{1}(0, \tau)\right|^{2}$ as a function of $\tau$ as computed from Eq. (70) and (b) antikink-shaped soliton $\left|q_{2}(0, \tau)\right|^{2}$ as computed from Eq. (71). (c) Chirping profile of soliton pair as a function of $\tau$ as computed from Eq. (72). The parameters are given in the text.

Figure $4 \mathrm{a}$ and $\mathrm{b}$ illustrate the intensity profiles of the above soliton pair for parameter values: $\alpha=1.6001, \gamma=$ $0.30814, \delta=0.15407, \eta=2, \Gamma=3, s=2.6885, \sigma=\frac{2}{3}$, $r=-2, \theta=-0.0074175, u=4.1184, \lambda=1.1841064$ and $k=-179.599$. Clearly, the profiles of the waves $q_{1}$ and $q_{2}$ represent the kink-shaped and anti-kink-shaped soliton pulses, respectively. These pulses may undistortedly propagate in normal-dispersion regime under the constraint $a_{2}>a_{3}$, which is obtainable for a positive GVD parameter satisfying the condition $\alpha>\frac{u \gamma(1-\eta)}{2(s-\sigma s-\lambda)}$. The corresponding chirp takes the expression:

$$
\delta \omega(\tau, z)=-2 p\left[\varphi^{2}+\kappa^{2} \tanh ^{2}(\mu \xi)\right]-q .
$$

Finally, Fig. 4c shows the pulse chirp obtained for the same values of parameters.

\subsection{Case $V$}

Next we consider bright-bright type solutions using the ansätze [31]:

$$
\begin{aligned}
& A(\xi)=\frac{C_{1}}{\sqrt{D_{1}+\cosh (\mu \xi)}}, \\
& B(\xi)=\frac{C_{2}}{\sqrt{D_{2}+\cosh (\mu \xi)}},
\end{aligned}
$$

where $C_{l}(l=1,2)$ represents the amplitude of the solitons for the two components $q_{1}$ and $q_{2}$, respectively, and $\mu$ is the inverse width of the solitons in both components. Substituting these ansätze into Eqs. (14) and (15) and setting the coefficients of the linearly independent functions to zero leads to

$$
\begin{aligned}
& -a_{6} C_{2}{ }^{4} D_{1}{ }^{2}-\frac{1}{4} D_{2}\left(4 a_{4} C_{1}{ }^{2} C_{2}{ }^{2} D_{1}+4 a_{1} C_{1}{ }^{4} D_{2}\right. \\
& -4 D_{1}\left(a_{3} C_{2}^{2} D_{1}+a_{2} C_{1}^{2} D_{2}-a_{6} D_{1} D_{2}\right) \\
& \left.+3 D_{2} \mu^{2}\right)=0 \text {, } \\
& -2 a_{6} C_{2}{ }^{4} D_{1}+a_{3} C_{2}^{2} D_{1}^{2}-2 a_{1} C_{1}^{2} D_{2}+2 a_{2} C_{1}^{2} D_{1} D_{2} \\
& +2 a_{3} C_{2}^{2} D_{1} D_{2}-2 a_{6} D_{1}^{2} D_{2}+a_{2} C_{1}^{2} D_{2}{ }^{2} \\
& -2 a_{6} D_{1} D_{2}{ }^{2}-a_{4} C_{1}{ }^{2} C_{2}{ }^{2}\left(D_{1}+D_{2}\right) \\
& -\frac{1}{2} D_{2}\left(3+D_{1} D_{2}\right) \mu^{2}=0, \\
& -a_{1} C_{1}{ }^{4}-a_{4} C_{1}{ }^{2} C_{2}{ }^{2}-a_{6} C_{2}{ }^{4}+a_{2} C_{1}{ }^{2} D_{1}+2 a_{3} C_{2}{ }^{2} D_{1} \\
& -a_{6} D_{1}^{2}+2 a_{2} C_{1}^{2} D_{2}+a_{3} C_{2}^{2} D_{2}-4 a_{6} D_{1} D_{2} \\
& -a_{6} D_{2}^{2}+\frac{1}{4}\left(-3-4 D_{1} D_{2}+D_{2}^{2}\right) \mu^{2}=0, \\
& a_{2} C_{1}^{2}+a_{3} C_{2}^{2}-2 a_{6}\left(D_{1}+D_{2}\right) \\
& +\frac{1}{2}\left(-D_{1}+D_{2}\right) \mu^{2}=0, \\
& -a_{1} C_{2}{ }^{4} D_{1}{ }^{2}-D_{2}\left\{C_{2}{ }^{2} D_{1}\left(a_{4} C_{1}{ }^{2}-a_{2} D_{1}\right)+\left[a_{6} C_{1}{ }^{4}\right.\right. \\
& \left.\left.+D_{1}\left(-a_{3} C_{1}^{2}+a_{6} D_{1}\right)\right] D_{2}\right\}-\frac{3 D_{1}^{2} \mu^{2}}{4}=0, \\
& -2 a_{1} C_{2}{ }^{4} D_{1}+a_{2} C_{2}{ }^{2} D_{1}{ }^{2}-2 a_{6} C_{1}{ }^{4} D_{2}+2 a_{3} C_{1}{ }^{2} D_{1} D_{2} \\
& +2 a_{2} C_{2}{ }^{2} D_{1} D_{2}-2 a_{6} D_{1}^{2} D_{2}+a_{3} C_{1}{ }^{2} D_{2}{ }^{2} \\
& -2 a_{6} D_{1} D_{2}^{2}-a_{4} C_{1}^{2} C_{2}^{2}\left(D_{1}+D_{2}\right)
\end{aligned}
$$




$$
\begin{aligned}
& -\frac{1}{2} D_{1}\left(3+D_{1} D_{2}\right) \mu^{2}=0, \\
- & a_{6} C_{1}^{4}-a_{4} C_{1}^{2} C_{2}^{2}-a_{1} C_{2}^{4}+a_{3} C_{1}^{2} D_{1} \\
& +2 a_{2} C_{2}^{2} D_{1}-a_{6} D_{1}^{2}+2 a_{3} C_{1}^{2} D_{2}+2 a_{2} C_{2}^{2} D_{2} \\
& -4 a_{6} D_{1} D_{2}-a_{6} D_{2}^{2} \\
& +\frac{1}{4}\left(-3+D_{1}^{2}-4 D_{1} D_{2}\right) \mu^{2}=0, \\
a_{3} C_{1}{ }^{2}+a_{2} C_{2}^{2}-2 a_{6}\left(D_{1}+D_{2}\right) & \\
& +\frac{1}{2}\left(D_{1}-D_{2}\right) \mu^{2}=0, \\
- & a_{6}+\frac{\mu^{2}}{4}=0 .
\end{aligned}
$$

By solving the above equations we can determine the solitons parameters as follows:

$$
\begin{aligned}
& \mu= \pm 2 \sqrt{a_{6}}, \\
& D_{2}=\frac{a_{3} C_{1}^{2}+a_{2} C_{2}^{2}}{4 a_{6}}, \\
& D_{1}=\frac{a_{2} C_{1}^{2}+a_{3} C_{2}^{2}}{4 a_{6}}, \\
& C_{2}=-C_{1} \sqrt{\frac{5 a_{2} a_{3}-2 a_{3}^{2}-8 a_{4} a_{6}+M / 2}{3 a_{2}^{2}-16 a_{1} a_{6}},}
\end{aligned}
$$

where $M^{2}=\left(-10 a_{2} a_{3}+4 a_{3}^{2}+16 a_{4} a_{6}\right)-4\left(-3 a_{2}^{2}+\right.$ $\left.16 a_{1} a_{6}\right)\left(48 a_{6}^{2}+4 a_{2} a_{3}-7 a_{3}^{2}+16 a_{6} a_{6}\right)$.

Substituting (73) and (74) into (5) and (6), one can obtain new chirped bright-bright soliton solutions for Eqs. (3) and (4) as

$$
\begin{aligned}
& q_{1}(z, \tau)=\frac{C_{1}}{\sqrt{D_{1}+\cosh (\mu(\tau-u z))}} \mathrm{e}^{\mathrm{i}(\chi(\xi)-k z),} \\
& q_{2}(z, \tau)=\frac{C_{2}}{\sqrt{D_{2}+\cosh (\mu(\tau-u z))}} \mathrm{e}^{\mathrm{i}(\chi(\xi)-k z),}
\end{aligned}
$$

which exist provided that $a_{6}>0$ and $\left(16 a_{1} a_{6}-3 a_{2}^{2}\right)\left[2\left(5 a_{2} a_{3}-2 a_{3}^{2}-8 a_{4} a_{6}\right)+M\right]<0$. In this case, the resulting intensity profiles will present the same shape as the ones derived in case III and the only difference concerns the maximum value of intensity which depends crucially on the pulse parameters and the conditions of existence of solutions.

Then substituting the above solutions into (12), we find that the corresponding chirping is given by

$$
\begin{aligned}
& \delta \omega(\tau, z)= \\
& \quad-p\left(\frac{C_{1}^{2}}{D_{1}+\cosh (\mu \xi)}+\frac{C_{2}^{2}}{D_{2}+\cosh (\mu \xi)}\right)-q .
\end{aligned}
$$

\section{Conclusion}

In this paper, we have investigated the propagation of chirped femtosecond solitons in a birefringent optical fiber that is described by two coupled nonlinear Schrödinger equations. The model used involves cubicquintic nonlinearity, self-steepening, self-frequency shift and the four-wave mixing term. After introducing a new ansatz that includes a novel form of chirping, the solutions were investigated within the framework of a set of coupled nonlinear ordinary differential equations involving many parameters. Various pairs of soliton solutions including bright-W shaped, kink and anti-kink, and bright-bright soliton pairs are derived, together with the nonlinear chirp that accompanied the existing pulses. The existence domain for the chirped soliton pair solutions has been found in the coupled NLS equations parameter set. This constitutes the first investigation of the existence of a variety of interesting soliton pair solutions with nonlinear chirping in birefringent fibers exhibiting higher-order effects. The obtained results are of interest, in particular, for possible chirped-soliton-based applications of highly birefringent fiber systems.

Future research problems for us include how to extend the proposed ansatz to find the nonlinear chirp that accompanied propagating soliton pulses for a set of $N$ coupled nonlinear Schrödinger-type equations. It also remains open how to find chirped solitons for coupled wave equations describing the interaction of a fundamental wave and its second harmonic in a quadratic nonlinear optical medium.

\section{Acknowledgments}

The work of third author DM is supported by the Ministry of Education, Sciences and Technological Development of Republic Serbia (grants III 44006 and TR 32051).

This research is funded by Qatar National Research Fund (QNRF) under the grant number NPRP 6-021-1005. The second and fourth authors (Anjan Biswas and Milivoj Belic) thank fully acknowledge this support from QNRF. The authors declare that there is no conflict of interest.

\section{References}

[1] G.P. Agrawal, Nonlinear Fiber Optics, Academic, New York 1995.

[2] A. Hesegawa, Y. Kodama, Solitons in Optical Communication, Oxford University Press, Oxford 1995.

[3] P.K. Shukla, B. Eliasson, Phys. Usp. 53, 51 (2010).

[4] F. Smirnov, Form Factors in Completely Integrable Models of Quantum Field Theory, World Sci., Singapore 1992.

[6] Min Li, Bo Tian, Wen-Jun Liu, Hai-Qiang Zhang, Pan Wang, Phys. Rev. E 81, 046606 (2010).

[6] Shaowu Zhang, Lin Yi, Phys. Rev. E 78, 026602 (2008).

[7] V.M. Vyas, P. Patel, P.K. Panigrahi, C.N. Kumar, W. Greiner, Phys. Rev. A 78, 021803 (R) (2008).

[8] K. Porsezian, B. Kalithasan, Chaos Solitons Fract. 31, 188 (2007).

[9] H. Triki, F. Azzouzi, Ph. Grelu, Opt. Commun. 309, 71 (2013).

[10] F. Azzouzi, H. Triki, Ph. Grelu, Appl. Math. Modell. 39, 1300 (2015). 
[11] H. Triki, A. Biswas, Math. Methods Appl. Sci. 34, 958 (2011).

[12] S.L. Palacios, A. Guinea, J.M. Fernández-Díaz, R.D. Crespo, Phys. Rev. E 60, R45 (1999).

[13] Yongsheng Tao, Jingsong He, Phys. Rev. E 85, 026601 (2012).

[14] Wei-Ping Zhong, M. Belić, Phys. Rev. E 82, 047601 (2010).

[15] N.Z. Petrović, M. Belić, Wei-Ping Zhong, Phys. Rev. E 83, 026604 (2011).

[16] Wen-Jun Liu, Bo Tian, Hai-Qiang Zhang, Tao Xu, He Li, Phys. Rev. A 79, 063810 (2009).

[17] A. Biswas, Phys. Lett. A 372, 5941 (2008).

[18] Z. Li, L. Li, H. Tian, G. Zhou, Phys. Rev. Lett. 84, 4096 (2000).

[19] A. Choudhuri, K. Porsezian, Opt. Commun. 285, 364 (2012).

[20] K.M. Spaulding, D.H. Yong, A.D. Kim, J.N. Kutz, J. Opt. Soc. Am. B 19, 1045 (2002).

[21] Xing Lü, Bo Tian, Phys. Rev. E 85, 026117 (2012).

[22] I.P. Kaminow, IEEE J. Quantum Electron. 17, 15 (1981).
[23] Alka, A. Goyal, R. Gupta, C.N. Kumar, T.S. Raju, Phys. Rev. A 84, 063830 (2011).

[24] C.N. Kumar, P. Durganandini, Pramana J. Phys. 53, 271 (1999).

[25] M. Desaix, L. Helczynski, D. Anderson, M. Lisak, Phys. Rev. E 65, 056602 (2002).

[26] V.I. Kruglov, A.C. Peacock, J.D. Harvey, Phys. Rev. Lett. 90, 113902 (2003).

[27] R. Radhakrishnan, A. Kundu, M. Lakshmanan, Phys. Rev. E 60, 3314 (1999).

[28] Pan Wang, Bo Tian, Opt. Commun. 285, 3567 (2012).

[29] Feng-Hua Qi, Bo Tian, Xing Lü, Rui Guo, YuShan Xue, Commun. Nonlin. Sci. Numer. Simulat. 17, 2372 (2012).

[30] A.H. Bhrawy, A.A. Alshaery, E.M. Hilal, M. Savescu, D. Milovic, K.R. Khan, M.F. Mahmood, Z. Jovanoski, A. Biswas, Optik 125, 4935 (2014).

[31] D. Milović, A. Biswas, Serb. J. Electr. Eng. 10, 365 (2013). 